\title{
Optical fiber-based handheld polarized photoacoustic computed tomography for detecting anisotropy of tissues
}

\author{
Yaguang Ren ${ }^{1 \#}$, Ying Zhang ${ }^{1,2 \#}$, Honghui $\mathrm{He}^{3}$, Liangjian Liu ${ }^{1}$, Xiaojun $\mathrm{Wu}^{2}$, Liang Song ${ }^{1}$, Chengbo Liu ${ }^{1 \wedge}$ \\ ${ }^{1}$ Research Laboratory for Biomedical Optics and Molecular Imaging, CAS Key Laboratory of Health Informatics, Shenzhen Institute of Advanced \\ Technology, Chinese Academy of Sciences, Shenzhen, China; ${ }^{2}$ School of Mechanical and Electrical Engineering, Xi'an University of Architecture \\ and Technology, Xi'an, China; ${ }^{3}$ Shenzhen Key Laboratory for Minimal Invasive Medical Technologies, Institute of Optical Imaging and Sensing, \\ Graduate School at Shenzhen, Tsinghua University, Shenzhen, China
}

Contributions: (I) Conception and design: Y Ren, Y Zhang; (II) Administrative support: X Wu, C Liu; (III) Provision of study materials or patients: H He, L Liu; (IV) Collection and assembly of data: Y Ren, Y Zhang; (V) Data analysis and interpretation: All authors; (VI) Manuscript writing: All authors; (VII) Final approval of manuscript: All authors.

\#These authors contributed equally to this work.

Correspondence to: Chengbo Liu. Shenzhen Institute of Advanced Technology, Chinese Academy of Sciences, 1068 Xueyuan Road, Shenzhen 518055 , China. Email: cb.liu@siat.ac.cn.

Background: Photoacoustic computed tomography (PACT) is a fast-developing biomedical imaging modality and has immense potential for clinical translation. It utilizes laser excitation and acoustic detection to achieve high spatial resolution and considerable imaging depth in biological tissues. Current PACT primarily treats the absorption coefficient of tissues as a scalar variable while reconstructing the image, which limits its use for anisotropic evaluation of the tissues. Thus, by incorporating polarized imaging methods to evaluate anisotropy, applications of PACT can be further enhanced. So far, dichroism-sensitive PACT has been suggested for polarization detection of biological tissues. However, this approach is unsuitable for intraoperative imaging, since high-power spatial light is needed for excitation, which is dangerous and inconvenient to operate. Thus, there is a need to develop a polarized PACT system suitable for clinical use.

Methods: Herein, we have proposed a specially designed handheld polarized PACT (HP-PACT) system, which was designed to promote intraoperative anisotropy detection of biological tissues. Excitation light was delivered by an optical fiber and reshaped by a compact set of lenses at the output end of the optical fiber. A polarizer was applied to generate linearly polarized light, and the polarization direction was adjusted by simply rotating the half-wave plate. Photoacoustic imaging (PAI) using excitation with several different polarization directions was carried out. Optical axes and the structure of the anisotropic objects were obtained using the principle of polarization detection with the PAI.

Results: We experimentally demonstrated the performance of HP-PACT by imaging both the polarized and unpolarized plastic films. The results showed that HP-PACT can successfully detect the direction of the optical axes of polarized plastic films and has the ability to image at different depths. When linearly polarized light with different polarization directions was used as excitation, PAI studies on a highly anisotropic bovine tendon and relatively low anisotropic mouse leg showed the structural differences between the 2 tissues. The quantified degrees of anisotropy of the bovine tendon and mouse legs were 0.6 and 0.3 , respectively.

Conclusions: The proposed HP-PACT is able to determine the anisotropic substances' optical axes and distinguish anisotropic substances from isotropic ones. Thus, HP-PACT has the potential for intraoperative diagnosis and treatment of anisotropic tissues, including nerves and tendons.

^ ORCID: Chengbo Liu, 0000-0001-7565-2044; Yaguang Ren, 0000-0001-9114-9596. 
Keywords: Photoacoustic computed tomography (PACT); polarization imaging; handheld

Submitted Jun 25, 2021. Accepted for publication Dec 14, 2021; Published online: 18 Jan 2022.

doi: 10.21037/qims-21-658

View this article at: https://dx.doi.org/10.21037/qims-21-658

\section{Introduction}

Photoacoustic computed tomography (PACT) utilizes laser excitation and acoustic detection to achieve high spatial resolution and considerable imaging depth in biological tissues $(1,2)$. Handheld PACT has shown to be a promising technique for clinical applications, especially intraoperative diagnosis or treatment (3-10). Usually, PACT assumes that the absorption coefficient of tissues is independent of polarization and thus uses unpolarized light for excitation. However, the absorption coefficients of most biological tissues are anisotropic (11), and the polarization information has not been fully exploited using traditional PACT. When polarization information is needed for clinical diagnosis or treatment, the conventional system cannot support it. For example, nerve and tendon surgeries usually result in severe damage, as it is often challenging to separate them from surrounding tissues. Consequently, the recipients of such surgeries consequently experience chronic pain or permanent paralysis. Tendons and nerves usually express anisotropy due to the highly orientated collagen fibers or microtubule arrangement of nerve axons and their alignment. Polarized PACT with linearly polarized light could identify nerves and tendons intraoperatively, which would be greatly beneficial to the surgery. Polarized PACT could also add new dimensional information to the imaging, with high potential for clinical application.

Polarized photoacoustic microscopy $(12,13)$ and dichroism-sensitive PACT (14) have been suggested for polarization detection of biological tissues. However, photoacoustic microscopy is unsuitable for the large field and deep tissue imaging needed during surgeries. Also, dichroism-sensitive PACT uses free-space light as an excitation, which is inconvenient to use during intraoperative imaging. Optical fiber-based PACT would be most suitable to achieve polarization imaging for intraoperative diagnosis or treatment. However, ordinary optical fibers cannot maintain the polarization state of light, and polarization-maintaining optical fibers cannot deliver high-power laser light due to the low-damage threshold for intraoperative photoacoustic imaging. In addition, the imaging probe should be handheld, lightweight, and easy to operate during intraoperative application. Thus, although it would help overcome challenges encountered in intraoperative diagnosis or treatment, it is challenging to adapt polarized PACT to the clinical setting.

In this study, we developed a handheld polarized PACT (HP-PACT) system to image the anisotropy of tissues. A multimode optical fiber was used to guide the excitation light to the probe, and a self-designed lens system integrated within the probe was used to reshape the output light to match the detection area of the ultrasound transducer. Although the multimode optical fiber may depolarize the excitation light, a polarizer inserted into the lens system was used to regenerate linearly polarized light, and the polarization direction was adjusted by rotating the half-wave plate mounted on the wave plate rotating frame. A theoretical analysis was conducted to extract the optical axis direction and structural information of the substance undergoing the test. We analyzed the imaging performance of HP-PACT with both polarized and unpolarized plastic films. We also analyzed the results of experimental studies conducted on highly anisotropic bovine tendons and relatively low anisotropic mouse legs using HP-PACT. The results showed that when mutually perpendicular polarized lights were used for excitation, structural differences between the 2 substances can be clearly distinguished. We present the following article in accordance with the MDAR checklist (available at https://qims.amegroups.com/article/ view/10.21037/qims-21-658/rc).

\section{Methods}

\section{Principle of polarization detection}

Generally, the photoacoustic signal is expressed as follows (15):

$$
P=\Gamma \eta \mu F
$$

where $\Gamma$ is the Gröneisen parameter, $F$ is the local optical fluence $\left(\mathrm{J} / \mathrm{cm}^{2}\right), \mu$ is the optical absorption coefficient 
of the biological tissue, and $\eta$ is the thermal conversion efficiency. For isotropic samples, $\mu$ is a constant. However, for anisotropic samples, the optical absorption coefficient $\mu$ of the biological tissue will be a variable changing with the angle between the optical axis and the polarization of the linearly polarized light $(\Delta \theta)$, and is expressed as follows (10):

$$
\mu(\Delta \theta)=\frac{\mu_{p}+\mu_{v}}{2}+\frac{\mu_{p}-\mu_{v}}{2} \cos (2 \Delta \theta)
$$

where $\mu_{p}$ and $\mu_{v}$ are the optical absorption coefficient when the polarization of the linearly polarized light is parallel or perpendicular to the optical axis of the biological tissue. Thus, the PA signal after combining [1] and [2] can be expressed as:

$$
P A=\Gamma \eta F\left(\frac{\mu_{p}+\mu_{v}}{2}+\frac{\mu_{p}-\mu_{v}}{2} \cos (2 \Delta \theta)\right)
$$

From Eq. [3], it is obvious that the PA intensity changes with the angle between the optical axis and the polarization of the linearly polarized light. In particular, when $\Delta \theta=0$ and $\Delta \theta=\pi / 2$, we will have the maximum $\left(P A_{\max }\right)$ and minimum photoacoustic intensities $\left(P A_{\min }\right)$, respectively:

$$
\left\{\begin{array}{l}
P A_{\max }=\Gamma \eta F \mu_{p} \\
P A_{\min }=\Gamma \eta F \mu_{v}
\end{array}\right.
$$

In addition, the direction of the optical axis can be calculated using the recorded PA intensity and the polarization direction of the incident light (10). Similarly, the structure of the specimen under the test can be extracted using by PA imaging using two different linearly polarized lights. The degree of linear polarization (DLP) can be defined as:

$$
D L P=\frac{P A_{\max }-P A_{\min }}{P A_{\max }+P A_{\min }}
$$

\section{Polarized photoacoustic probe}

During the intraoperative procedure, a linear-array ultrasound transducer is typically used. Thus, while imaging, it is necessary to reshape the light spot at the output end of the fiber into a shape that matches the detection area of the ultrasound traducer. Also, a uniform light spot is preferred for high-quality imaging. We first performed optical simulation using ZEMAX software (Radiant Vision Systems Corporation, Redmond, WA, USA) to achieve a $3 \mathrm{~mm} \times 10 \mathrm{~mm}$ slender beam at the surface of the imaging sample to match the detection region of the linear-array ultrasound transducer. The parameters of the multimode optical fiber [core diameter 1,500 um, numerical arperture (NA) 0.22], the spot size, and the parameters of the plano-convex cylindrical lenses were set as per their real values. The distances between each optical element were set to vary in the ZEMAX simulation. The global optimization was performed by adjusting these variables to achieve the required beam size and uniform illumination at the surface of the imaging plane (approximately $8 \mathrm{~mm}$ below the ultrasound transducer). The sketch of the ZEMAX simulation model is shown in Figure 1, in which the illumination light is reshaped into a slender beam using 4 plano-convex cylindrical lenses (the lenses from top to bottom are LJ1942L2-B, LJ638L1-B, LJ1105L1, and LJ1125L1, Thorlabs, Newton, NJ, USA).

For realizing polarization detection, a polarizer (extinction ratio >1,000:1, LPNIRB050, Thorlabs) was used to generate linearly polarized light at the output end of the optical fiber. A half-wave plate (HWP; WPA2420-6501100, Union Optic, Sindos, Greece) mounted on an angleadjustable frame was used to easily adjust the excitation light's polarization orientation. Both optical components were inserted between the LJ1105L1 and LJ1125L1 lenses (Figure 1) to achieve a compact and lightweight polarized photoacoustic probe for intraoperative imaging. The weight of the probe is only about $375 \mathrm{~g}$, which facilitates intraoperative use.

\section{HP-PACT experimental setup}

The HP-PACT system (Figure 2) presented in this study is the enhanced version of the system presented in our previous work (16) with multiple differences compared with other fiber-based PACT systems. Firstly, a polarizer was added in the lens group to generate linearly polarized light and achieve anisotropic imaging. Furthermore, a uniform slender beam was designed based on ZEMAX simulation to achieve a uniform light spot at the surface of the imaging sample as well as to match the detection region of the lineararray ultrasound transducer. Additionally, a HWP mounted on an angle-adjustable frame mounting and a specially designed probe housing with a small opening (i.e., hole) allowed the visualization of the polarization value to appear on the angle-adjustable frame of the HWP. This is also very important for ease of intraoperative handling. With this system, an optical parametric oscillator (OPO) laser source (Innolas GmbH, Bonn, Germany) working at $780 \mathrm{~nm}$ with a $30 \mathrm{~Hz}$ pulse repetition rate was coupled to a multimode 


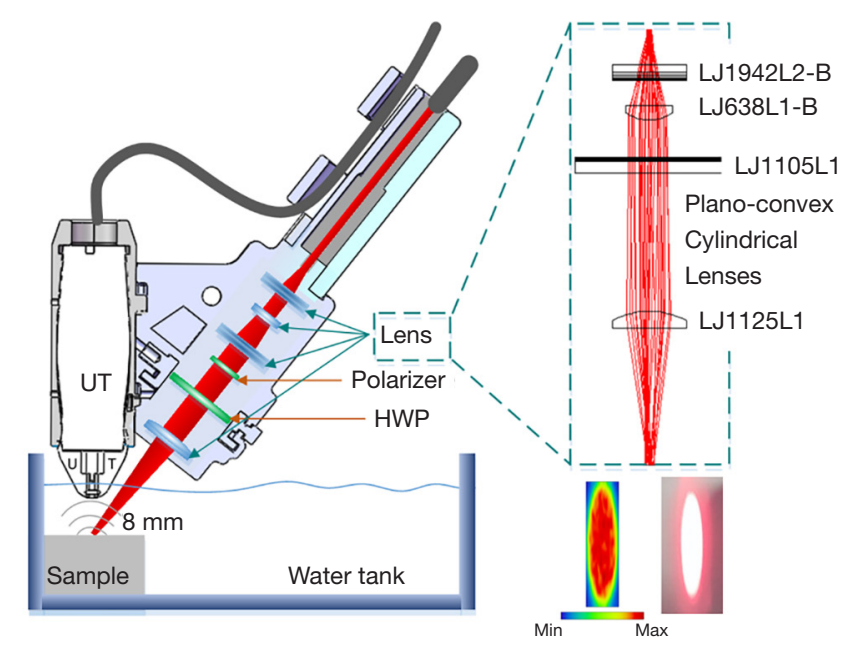

Figure 1 Handheld polarized photoacoustic probe. UT, ultrasound transducer; HWP, half-wave plate.

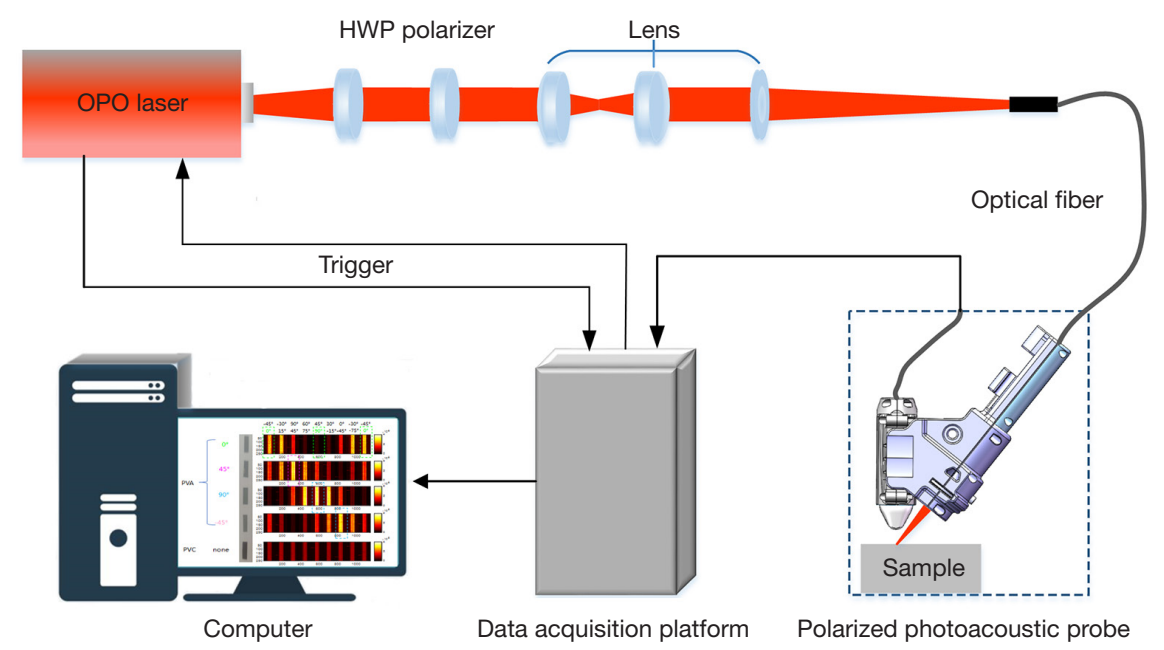

Figure 2 Experimental setup of HP-PACT system. HP-PACT, handheld polarized photoacoustic computed tomography; HWP, half-wave plate.

optical fiber. The coupling efficiency of the optical fiber was almost $91 \%$, on top of which the pano-convex cylindrical lenses used for light reshaping led to another $10 \%$ optical loss. Furthermore, only almost $50 \%$ of light could pass through the polarizer and become linearly polarized light. Thus, the overall light efficiency of the proposed probe was approximately $41 \%$. A customized 128-element linear-array ultrasound transducer [center frequency : $15 \mathrm{MHz} \pm 10 \%$, bandwidth: $>60 \%$ (at $-6 \mathrm{~dB}$ ), Vermon, Tours, France] was used to receive the ultrasound signals. A Vantage 128 research ultrasound platform (Verasonics Inc., Kirkland, WA, USA) synchronized with the excitation laser was used for photoacoustic data acquisition.

\section{Polarization calibration of the linearly polarized light}

The calibration of the polarized photoacoustic probe was conducted before PAI experiments to achieve accurate polarization detection. A collimated continuous light source (wavelength: $650 \mathrm{~nm}$, spot diameter: $5 \mathrm{~mm}$, and 

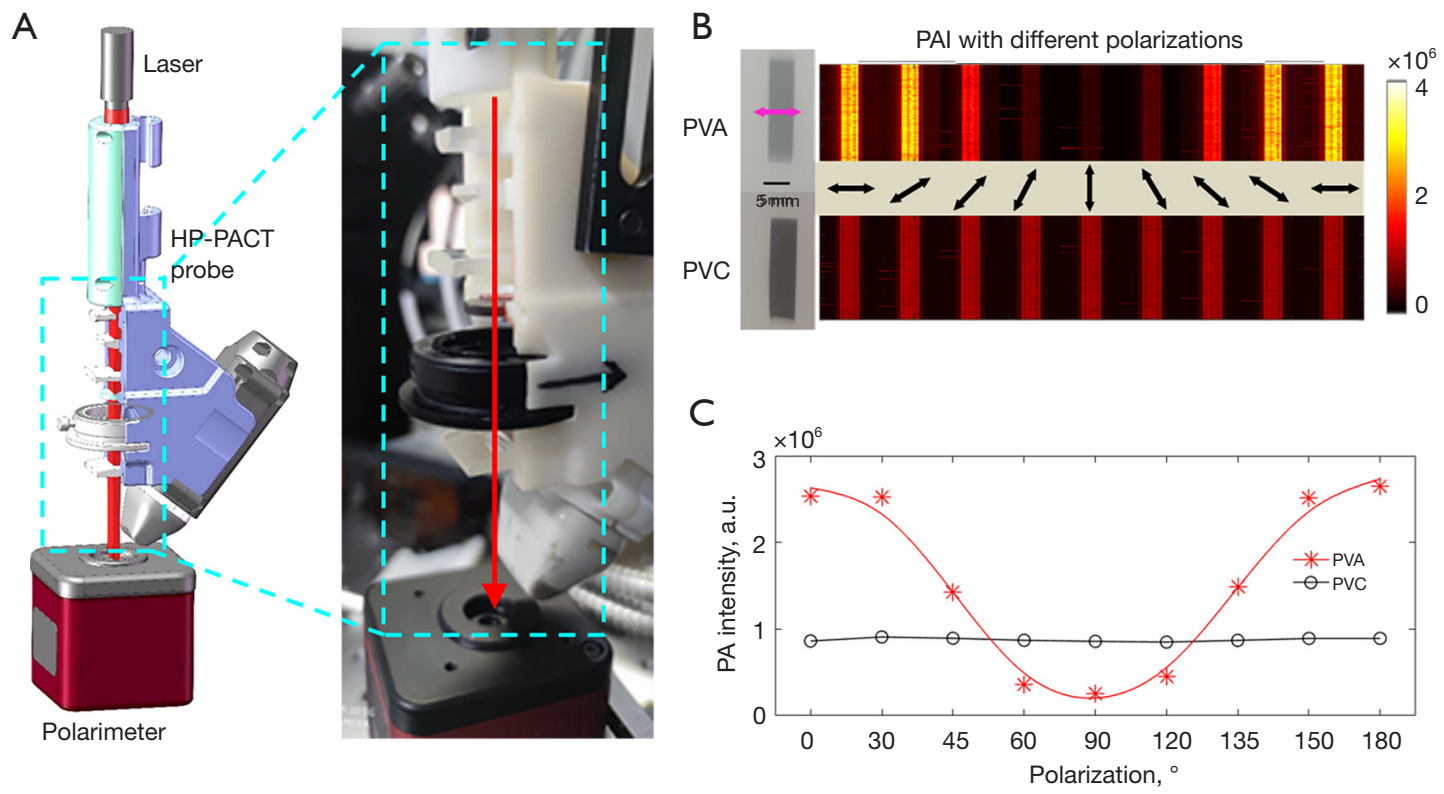

Figure 3 Polarization calibration of linearly polarized light. (A) Photograph of the calibration setup. (B) PAI of the PVA and PVC with different polarizations. (C) The photoacoustic intensity of PVA and PVC is shown in (B). HP-PACT, handheld polarized photoacoustic computed tomography; PAI, photoacoustic imaging; PVA, polyvinyl alcohol; PVC, polyvinyl chloride.

Table 1 Polarization calibration results of linearly polarized light (values shown on the adjustable frame of the HWP at different polarization directions)

\begin{tabular}{lccccccccc}
\hline Polarization & $0^{\circ}$ & $30^{\circ}$ & $45^{\circ}$ & $60^{\circ}$ & $90^{\circ}$ & $120^{\circ}$ & $135^{\circ}$ & $150^{\circ}$ & $180^{\circ}$ \\
\hline HWP & 341.5 & 326.5 & 319 & 311.5 & 296.5 & 281.5 & 274.0 & 266.5 & 251.5 \\
\hline
\end{tabular}

HWP, half-wave plate.

maximum power: $5 \mathrm{~mW}$, Taizhu Technology Co., Ltd., Shenzhen, China) was used for the polarization calibration. A polarimeter (PAX1000VIS/M, 400-700 nm, Thorlabs) was used to measure the polarization direction of the output light. The collimated light source, handheld PAI probe, and polarimeter were fixed as shown in Figure 3 to ensure that the incident light spot was vertical to the polarimeter. The small opening at the right side of the probe housing allowed visualization of values appearing on the angle-adjustable frame of the HWP. The HWP was rotated to get the linearly polarized light at $0^{\circ}, 30^{\circ}, 45^{\circ}, 60^{\circ}, 90^{\circ}, 120^{\circ}, 135^{\circ}$, $150^{\circ}$, and $180^{\circ}$. The calibration results are shown in Table 1 (multiple measurement error $< \pm 0.5^{\circ}$ ).

The PAI of polarized and unpolarized plastic films (Jiateng Film and Television Equipment Co. Ltd, Xuzhou, China) immersed in scattering medium (1\% agar with $1 \%$ intralipid) was carried out using polarized light with different polarizations. The main component of polarized plastic film is polyvinyl alcohol (PVA), where the iodine molecules are arranged along a straight line to form a long chain resulting in good dichroism. The main component of unpolarized plastic film is polyvinyl chloride (PVC), which has no dichroism. In Figure 3B, the purple arrow represents the polarizer's optical axis, and the black arrow represents the polarization direction of the incident laser light. The photoacoustic signal intensities of PVA showed periodic changes in sync with the changing of the polarization excitation light. However, no changes were found for the PVC material with the change in polarization, see Figure $3 B, 3 C$. When the polarization direction of the incident light was parallel to the optical axis of PVA, the strongest photoacoustic signal intensity was obtained. Conversely, when the polarization direction of the incident light was $90^{\circ}$, the weakest photoacoustic signal intensity was detected. The results demonstrated in Figure $3 B, 3 C$ are consistent with the theoretical analysis (Eqs. $[3,4]$ ), thus verifying 


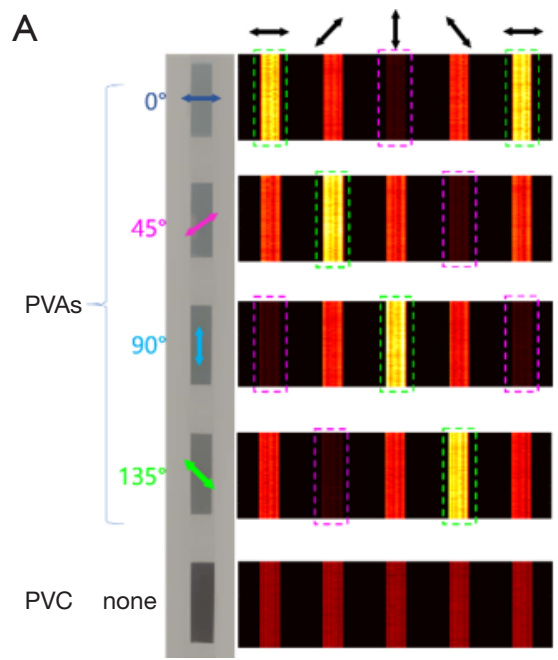

B

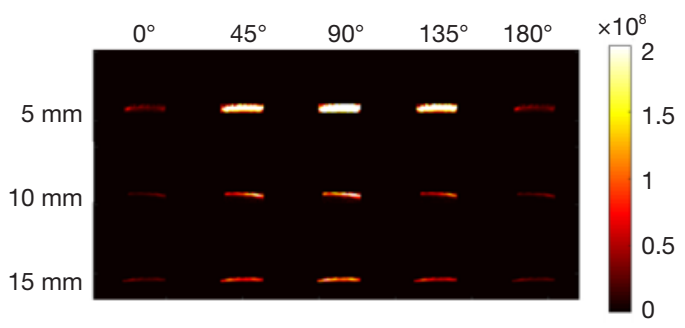

Figure 4 PVA with different polarizing directions and PVC were imaged using HP-PACT with 5 polarization directions. PVAs, polyvinyl alcohols; PVC, polyvinyl chloride; HP-PACT, handheld polarized photoacoustic computed tomography.

the calibration. The calibrated HP-PACT could be used successfully to detect the polarized substance by simply rotating the HWP.

\section{Results}

\section{Phantom experimental results}

To verify that the optical axis of the polarized substances can be determined, and polarized and unpolarized substances can be differentiated using the proposed HP-PACT system, we further imaged 4 PVAs with different polarization directions $\left(0^{\circ}, 45^{\circ}, 90^{\circ}\right.$, and $\left.135^{\circ}\right)$ and $\mathrm{PVC}$, as shown in Figure $4 A$. When the polarization direction of the incident light was changed manually, the photoacoustic intensities also changed, corresponding to the change in angle between the optical axis of PVA and the polarization directions of the incident light. The green and purple dotted rectangles cover the maximum and minimum photoacoustic signal regions, respectively, indicating the optical axis of PVAs. For PVC, similar to the calibration results shown in Figure 3, the photoacoustic intensity remained constant. Thus, it is easy to distinguish between polarized PVAs and unpolarized PVC. In addition, results in Figure $4 A$ show that the proposed HP-PACT has high fidelity and stability for optical axis determination.

We also imaged PVAs in scattering media at different depths ( $5 \mathrm{~mm}, 10 \mathrm{~mm}$, and $15 \mathrm{~mm}$ ) using linearly polarized light with different polarization directions to show that our method can also resolve deep polarized substances, and the results are shown in Figure 4B. In addition, the DLP of the incident light beam at different depths was also evaluated using the photoacoustic intensity (Eq. [5]), and they were $0.92,0.79$, and 0.67 , at $5 \mathrm{~mm}, 10 \mathrm{~mm}$, and $15 \mathrm{~mm}$, respectively.

\section{Tissue experimental results}

To show the feasibility of HP-PACT for biological tissue imaging, we obtained PAI of an ex vivo bovine tendon (Figure 5 lower), a typical anisotropic tissue having a high concentration of collagen, and PAI of a mouse leg, see Figure 5 (upper), which is a relatively low anisotropic tissue. The $780 \mathrm{~nm}$ laser fluence at the surface of the biological tissues is approximately $20 \mathrm{~mJ} / \mathrm{cm}^{2}$, well below the American National Standards Institute safety standard limit (17). Regions marked by a dotted rectangular box in Figure $5 \mathrm{~A}$ were measured 10 times to exclude influence from external factors (18) such as random white noise, intrinsic noise from devices (e.g., ultrasound transducer and ultrasonic data acquisition platform), background noise, and so on. All measurements were averaged, and the resultant photoacoustic images from these measurements are shown in Figure 5B. The yellow arrows indicate the polarization direction of the excitation light used in PAI. Subtractions were conducted in Figure $5 B$ using the 2 images obtained with different polarization lights. In 


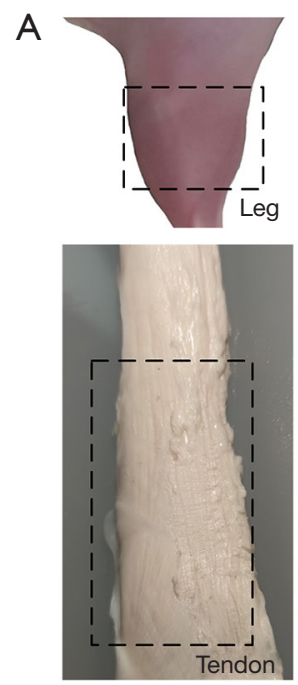

\section{B}
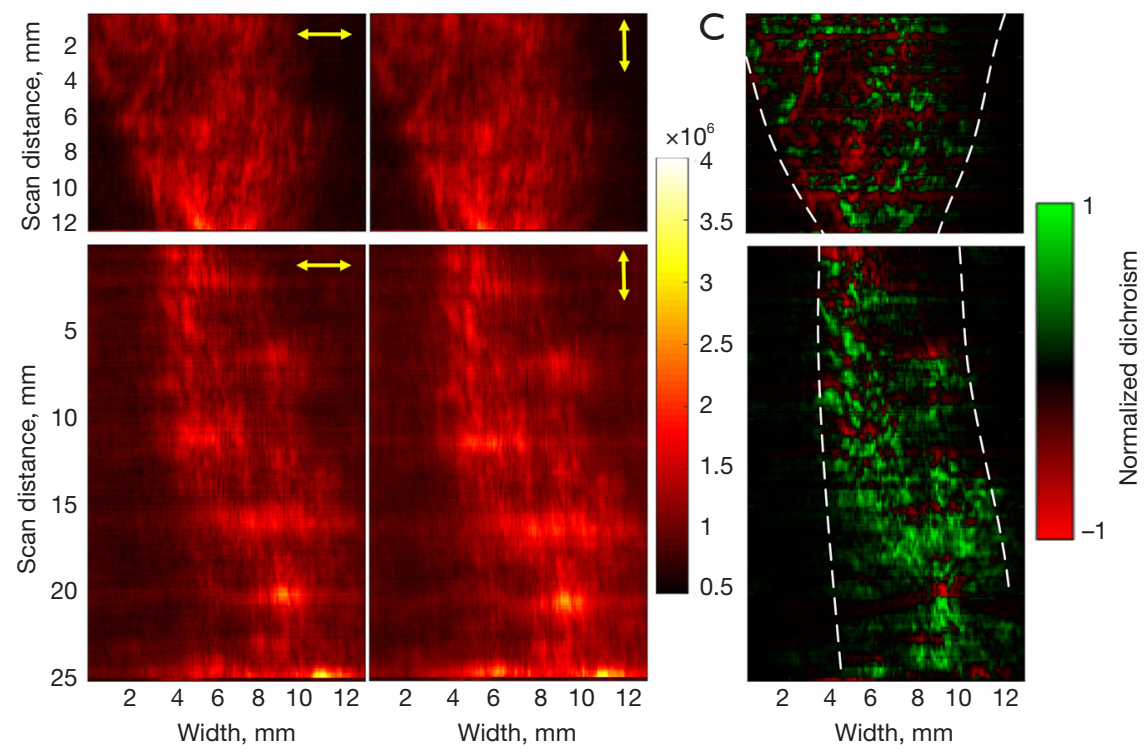

Figure 5 HP-PACT images of a mouse leg and ex vivo bovine tendon. (A) Photograph of the mouse leg (upper) and bovine tendon (lower). (B) HP-PACT images of mouse leg (upper) and bovine tendon (lower) with the polarization of the excitation light being $0^{\circ}$ (horizontal) and $90^{\circ}$ (vertical). (C) Extracted structure of the mouse leg (upper) and bovine tendon (lower) to be obtained by subtracting the 2 photoacoustic images with different polarizations in (B). HP-PACT, handheld polarized photoacoustic computed tomography.

particular, the photoacoustic image of $0^{\circ}$ polarized light was subtracted from the image of $90^{\circ}$ polarized light. The resulting image, shown in Figure 5C, was color-coded based on the subtracted values. All pixels with positive values were colored-coded in green, and all pixels with negative values were color-coded in red. As can be seen from Figure 5 C, the bovine tendon image is predominantly green, indicating that it had a high anisotropic property. In contrast, the mouse leg [a specific-pathogen-free (SPF) BALB/c mouse male, 6 weeks old, 18-20 g, from Beijing Vital River Laboratory Animal Technology Co., Ltd., Beijing, China] image shows randomly distributed green and red color, indicating it had low anisotropic properties. Moreover, the degrees of anisotropy of both tendon and mouse leg were quantified according to equation 5 , and were 0.6 and 0.3 , respectively. This finding further confirmed our hypothesis that the HP-PACT has the capability for anisotropic tissue identification. Nevertheless, it should be noted that due to the different biological molecular arrangements between the polarized plastic films and biological tissues (19), the changes in the PAI with variable polarization were not as significant in biological tissues as compared to the polarized plastic film. All animal procedures were performed under a project license (No. SIAT-IACUC-210705-YGSRYG-A1975) granted by the Animal Ethics Committee of Shenzhen Institutes of Advanced Technology, Chinese Academy of Sciences, in compliance with the national guidelines for the care and use of animals.

\section{Discussion and conclusions}

In this study, we established an HP-PACT system to meet the challenges of anisotropy detection of tissues. This system is compact and utilizes a portable photoacoustic probe for imaging. Compared with the previously proposed laser diode and light-emitting diode (LED)-based PAI system $(20,21)$, the proposed HP-PACT can provide higher pulse energy to allow a higher signal-to-noise ratio (SNR) and larger penetration depth. Laser diode- or LED-based systems, however, have the advantages of lower cost and footprint. The phantom results confirmed that the proposed HP-PACT could determine the optical axis and extract the structure of anisotropic substances during the test. The photoacoustic intensity changes in the phantom were clearly observable when linearly polarized lights with different polarized directions were applied. Moreover, compared to those of the mouse leg, photoacoustic images of the bovine tendon showed more significant signal variations, which confirmed that HP-PACT can be used to distinguish the anisotropic substances from the low anisotropic or 
isotropic ones in biological tissues. Since the polarization detection has advantages in suppressing background noise, it can acquire detailed features and identify weak targets that cannot be seen by the conventional PAI method. In in vivo applications, respiratory motion is known to result in image blurring and loss of resolution. However, respiratory gating is a mature technology used for respiratory motion removal. So far, this technology has been well developed in photoacoustic tomography (22). Other efforts to correct breathing motion include postprocessing algorithms $(23,24)$. In addition, the imaging speed can be further improved with a fast laser source to reduce the breathing artifact. Moreover, HP-PACT can be combined with another polarized sensitive imaging system, for example, polarized sensitive optical coherence tomography (PSOCT) to further improve sensing capability (25). For HP-PACT, polarization information of biological tissues is detected based on the absorption difference between different polarization excitations. For PS-OCT, it is based on the principle of low-coherence interferometry, where a low-coherence polarized light beam is directed onto the targeted tissue. The backscattered light contains tissue polarization information and interferes with a reference beam splitting off from the original light beam. Therefore, HP-PACT provides rich and unique molecular information based on optical absorption contrast, whereas PS-OCT offers structural information based on optical scattering contrast. The 2 methods naturally complement each other in terms of contrast mechanism. In conclusion, the proposed portable probe and easy-to-use system presented in this study have the potential to be used in diagnosis and treatment of many clinical diseases, including tendon repair, removal of neuroangioma, malignant tumor identification, and eye diseases.

\section{Acknowledgments}

Funding: This work was supported by the National Natural Science Foundation of China (No. 92059108, No. 81927807); Chinese Academy of Sciences (No. YJKYYQ20190078, No. GJJSTD20210003); Chinese Academy of Sciences Youth Innovation Promotion Association (No. 2019352); Shenzhen Science and Technology Innovation Grant (No. JCYJ20200109141222892); National Key R\&D Program of China (No. 2020YFA0908800); Chinese Academy of Sciences Key Laboratory of Health Informatics (No. 2011DP173015); Guangdong Provincial Key Laboratory of Biomedical Optical Imaging (No. 2020B121201010); Shenzhen Key Laboratory of Molecular Imaging (No. ZDSY20130401165820357); Natural Science Foundation of Shenzhen (No. JCYJ20190806150001764); and the Natural Science Foundation of Guangdong Province (No. 2020A1515010978).

\section{Footnote}

Reporting Checklist: The authors have completed the MDAR checklist. Available at https://qims.amegroups.com/article/ view/10.21037/qims-21-658/rc

Conflicts of Interest: All authors have completed the ICMJE uniform disclosure form (available at https://qims. amegroups.com/article/view/10.21037/qims-21-658/coif). The authors have no conflicts of interest to declare.

Ethical Statement: The authors are accountable for all aspects of the work in ensuring that questions related to the accuracy or integrity of any part of the work are appropriately investigated and resolved. All animal procedures were performed under a project license (No. SIAT-IACUC-210705-YGS-RYG-A1975) granted by the Animal Ethics Committee of Shenzhen Institutes of Advanced Technology, Chinese Academy of Sciences, in compliance with the national guidelines for the care and use of animals.

Open Access Statement: This is an Open Access article distributed in accordance with the Creative Commons Attribution-NonCommercial-NoDerivs 4.0 International License (CC BY-NC-ND 4.0), which permits the noncommercial replication and distribution of the article with the strict proviso that no changes or edits are made and the original work is properly cited (including links to both the formal publication through the relevant DOI and the license). See: https://creativecommons.org/licenses/by-nc-nd/4.0/.

\section{References}

1. Yao J, Kaberniuk AA, Li L, Shcherbakova DM, Zhang R, Wang L, Li G, Verkhusha VV, Wang LV. Multiscale photoacoustic tomography using reversibly switchable bacterial phytochrome as a near-infrared photochromic probe. Nat Methods 2016;13:67-73.

2. Zhang C, Zhao H, Xu S, Chen N, Li K, Jiang X, Liu L, Liu Z, Wang L, Wong KKY, Zou J, Liu C, Song L. 
Multiscale high-speed photoacoustic microscopy based on free-space light transmission and a MEMS scanning mirror. Opt Lett 2020;45:4312-5.

3. Wang $\mathrm{LV}, \mathrm{Hu} \mathrm{S}$. Photoacoustic tomography: in vivo imaging from organelles to organs. Science 2012;335:1458-62.

4. Steinberg I, Huland DM, Vermesh O, Frostig HE, Tummers WS, Gambhir SS. Photoacoustic clinical imaging. Photoacoustics 2019;14:77-98.

5. Kitai T, Torii M, Sugie T, Kanao S, Mikami Y, Shiina T, Toi M. Photoacoustic mammography: initial clinical results. Breast Cancer 2014;21:146-53.

6. Choi W, Park EY, Jeon S, Kim C. Clinical photoacoustic imaging platforms. Biomed Eng Lett 2018;8:139-55.

7. Brown E, Brunker J, Bohndiek SE. Photoacoustic imaging as a tool to probe the tumour microenvironment. Dis Model Mech 2019;12:dmm039636.

8. Kim J, Park EY, Park B, Choi W, Lee KJ, Kim C. Towards clinical photoacoustic and ultrasound imaging: Probe improvement and real-time graphical user interface. Exp Biol Med (Maywood) 2020;245:321-9.

9. Wang R, Pan T, Huang L, Liao C, Li Q, Jiang H, Yang J. Photoacoustic imaging in evaluating early intestinal ischemia injury and reperfusion injury in rat models. Quant Imaging Med Surg 2021;11:2968-79.

10. Qiu T, Lan Y, Gao W, Zhou M, Liu S, Huang W, Zeng S, Pathak JL, Yang B, Zhang J. Photoacoustic imaging as a highly efficient and precise imaging strategy for the evaluation of brain diseases. Quant Imaging Med Surg 2021;11:2169-86.

11. Jacques SL. Optical properties of biological tissues: a review. Phys Med Biol 2013;58:R37-61.

12. Zhang Z, Shi Y, Xiang L, Xing D. Polarized photoacoustic microscopy for vectorial-absorption-based anisotropy detection. Opt Lett 2018;43:5267-70.

13. Zhou Y, Chen J, Liu C, Liu C, Lai P, Wang L. Singleshot linear dichroism optical-resolution photoacoustic microscopy. Photoacoustics 2019;16:100148.

14. Qu Y, Li L, Shen Y, Wei X, Wong TTW, Hu P, Yao J, Maslov K, Wang LV. Dichroism-sensitive photoacoustic computed tomography. Optica 2018;5:495-501.

15. Xia J, Yao J, Wang LV. Photoacoustic tomography: principles and advances. Electromagn Waves (Camb) 2014;147:1-22.

16. Ren Y, Sedgwick AC, Chen J, Thiabaud G, Chau CV, An J, Arambula JF, He XP, Kim JS, Sessler JL, Liu C. Manganese(II) Texaphyrin: A Paramagnetic Photoacoustic
Contrast Agent Activated by Near-IR Light. J Am Chem Soc 2020;142:16156-60.

17. Laser Institute of America, American National Standard for Safe Use of Lasers ANSI Z136.1-2000, American National Standards Institute, Inc., New York, NY, 2000.

18. Li J, Yu B, Zhao W, Chen W. A review of signal enhancement and noise reduction techniques for tunable diode laser absorption spectroscopy. Appl Spectrosc Rev 2014;49:666-91.

19. Silva MM, Cyster LA, Barry JJ, Yang XB, Oreffo RO, Grant DM, Scotchford CA, Howdle SM, Shakesheff KM, Rose FR. The effect of anisotropic architecture on cell and tissue infiltration into tissue engineering scaffolds. Biomaterials 2006;27:5909-17.

20. Kuniyil Ajith Singh M, Xia W. Portable and Affordable Light Source-Based Photoacoustic Tomography. Sensors (Basel) 2020;20:6173.

21. Agrawal S, Fadden C, Dangi A, Yang X, Albahrani H, Frings N, Heidari Zadi S, Kothapalli SR. Light-EmittingDiode-Based Multispectral Photoacoustic Computed Tomography System. Sensors (Basel) 2019;19:4861.

22. Xia J, Chen W, Maslov K, Anastasio MA, Wang LV. Retrospective respiration-gated whole-body photoacoustic computed tomography of mice. J Biomed Opt 2014;19:16003.

23. Attia ABE, Balasundaram G, Moothanchery M, Dinish US, Bi R, Ntziachristos V, Olivo M. A review of clinical photoacoustic imaging: Current and future trends. Photoacoustics 2019;16:100144.

24. Erlöv T, Sheikh R, Dahlstrand U, Albinsson J, Malmsjö M, Cinthio M. Regional motion correction for in vivo photoacoustic imaging in humans using interleaved ultrasound images. Biomedical Optics Express 2021;12:3312-22.

25. Hosseinaee Z, Nima Abbasi, Pellegrino N, Khalili L, Mukhangaliyeva L, Haji Reza P. Functional and structural ophthalmic imaging using noncontact multimodal photoacoustic remote sensing microscopy and optical coherence tomography. Sci Rep 2021;11:11466.

Cite this article as: Ren Y, Zhang Y, He H, Liu L, Wu X, Song L, Liu C. Optical fiber-based handheld polarized photoacoustic computed tomography for detecting anisotropy of tissues. Quant Imaging Med Surg 2022;12(4):2238-2246. doi: 10.21037/qims-21-658 